Case Report

\title{
Solving a Class of Nonlinear Delay Integro-differential Equations by Using Differential Transformation Method
}

\author{
Mohammad Bagher Moghimi, Abdollah Borhanifar \\ Faculty of Mathematical Sciences, University of Mohaghegh Ardabili, Ardabil, Iran \\ Email address: \\ moghimi@uma.ac.ir(M. B. Moghimi),borhani@uma.ac.ir (A. Borhanifar)
}

\section{To cite this article:}

Mohammad Bagher Moghimi, Abdollah Borhanifar. Solving a Class of Nonlinear Delay Integro-differential Equations by Using Differential Transformation Method. Applied and Computational Mathematics. Vol. 5, No. 3, 2016, pp. 142-149. doi: 10.11648/j.acm.20160503.18

Received: April 30, 2016; Accepted: June 14, 2016; Published: July 13, 2016

\begin{abstract}
In this paper, differential transformation method is used to find exact solutions of nonlinear delay integrodifferential equations. Many theorems are presented that required for applying differential transformation method for nonlinear delay integro-differential equation. The validity and efficiency of the proposed method are demonstrated through several tests.
\end{abstract}

Keywords: Delay Integro-differential Equation, Delay Differential Equation, Differential Transformation Method, Closed Form Solution

\section{Introduction}

Finding the analytical solutions of functional equations has been devoted attention of mathematicians's interest in recent years. Several methods are proposed to achieve this purpose, such as [1]-[5]. The nonlinear integro-differential equations with variable delays reads

$$
\begin{gathered}
y^{\prime}(t)=f\left(t, y(t), y\left(q_{0} t\right), y\left(t-\tau_{0}\right), \int_{t_{0}}^{t} K\left(t, s, y(s), y\left(q_{1} s\right), y(s-\right.\right. \\
\left.\left.\left.\tau_{1}\right)\right) d s\right), t \in I=\left[t_{0}, T\right],
\end{gathered}
$$

subject to initial condition

$$
y(t)=\phi(t), t \in\left[-\tilde{\tau}, t_{0}\right], \tilde{\tau}=\min \left\{\tau_{0}, \tau_{1}\right\},
$$

where $f$ and $\phi$ are analytical function and the nonlinear kernel $K$ is continuous on the domain

$$
D=\{(t, s): t-\tilde{\tau} \leq s \leq t, t \in I\},
$$

Here $q_{i} \in(0,1)$, for $i=0,1$, are the coefficients of vanishing delay function $\theta(t)=q_{i} t$, which is used in the pantograph equation, and $\tau_{i}>0$, for $i=0,1$, are the constants of non-vanishing delay $\theta(t)=t-\tau_{i}$, that is a classical case of delay functions.

This type of equations have widely occurred in many biological and control problems (see $[6,7]$ and their references therein). As we know, much work has been done on developing and analyzing numerical methods for solving onedimensional integral and integro-differential equations without delay. But in delay cases, a small amount of work has been done. Delay integro-differential equations are usually difficult to solve analytically so it is required to obtain an efficient approximate solution. Therefore, they have been of great interest by several authors. In literature, there exist few numerical techniques applied to solving delay integrodifferential equation. The main of these literatures carried out by Hermann Burnner, which most of the recent studies belong to him [8]-[14]. Asymptotic error expansions for linear multistep methods in [15] and Stability properties of a scheme for the approximate solution in [16] of a delay-integro-differential equation have been discussed by Baker and Ford, Brunner in [6], Brunner and et al in [12] applied a numerical method based on finite difference method and a collocation method to solving (1), and Koto in $[17,18]$ and W. H. Enright and Hu in [19] used Runge-Kutta method and $\theta$-method to solving Eq. (1), respectively. In recent years, many papers have also studied the convergence and stability of numerical methods for (1) or equations of more general forms. For the convergence of numerical methods, we refer to [8]-[12], and for the stability we refer to [13]-[18] and their references therein.

The method that is developed in this paper depends on DTM, introduced by Borhanifar [20, 21] for solution of linear and nonlinear problems and Zhou [22] in a study about 
electrical circuits. It is a semi-numerical-analytical technique that formulates Taylor series in a totally different manner. With this technique, the given differential equation and related initial conditions are transformed into a recurrence equation that finally leads to the solution of a system of algebraic equations as coefficients of a power series solution. This method is useful for obtaining exact and approximate solutions of linear and nonlinear differential equations. There is no need for linearization or perturbations, large computational work and round-off errors are avoided. It has been used to solve effectively, easily and accurately a large class of linear and nonlinear problems with approximations. It is possible to solve system of differential equations [23, 24, 25], differential-algebraic equations [26], difference equations [27], differential difference equations [28], partial differential equations [29, 30, 31, 32], Nonlinear partial differential equations [33], fractional differential equations $[34,35]$, time-fractional diffusion equation [36], pantograph equations [37], vibration problems of circular Euler-Bernoulli beams [38], Axisymmetric vibrations and buckling analysis [39], projectile motion in a quadratic resistant medium [40], one- dimensional Volterra integral and integro-differential equations [41, 42, 43] by using this method. In this work, we will extend one-dimensional differential transformation method (DTM), by presenting and proving some new theorems, to solve a class of nonlinear delay integrodifferential equation which it's kernel function is also involve delay (vanishing and non-vanishing delays).

The remainder of this paper is organized as follows: The structure of differential transformation method is stated in
Section 2. Hereby, some theorems are presented and proved. In Section 3, the proposed method is applied to solve several delay integro-differential equations. A conclusion is presented in Section 4.

\section{Basic Idea of Differential Transformation Method}

In this section, the basic definitions of differential transformation are introduced as follows:

An arbitrary function $u(t)$ that is analytical function can be expanded in Taylor series about a point $t=0$ as

$$
y(t)=\sum_{k=0}^{\infty} \frac{\left(t-t_{0}\right)^{k}}{k !}\left[\frac{d^{k} y(t)}{d t^{k}}\right]_{t=t_{0}}
$$

If $Y(k)$ is defined as

$$
\mathrm{Y}(k)=\frac{1}{k !}\left[\frac{d^{k} y(t)}{d t^{k}}\right]_{t=t_{0}}, \text { where } k=0,1, \ldots, \infty
$$

then Eq. (3) reduces to

$$
\mathrm{y}(\mathrm{t})=\sum_{\mathrm{k}=0}^{\infty} \mathrm{Y}(\mathrm{k})\left(\mathrm{t}-\mathrm{t}_{0}\right)^{\mathrm{k}} .
$$

The $\mathrm{Y}(\mathrm{k})$ defined in Eq. (4), is called the differential transform of function $y(t)$. The following theorems that can be deduced from Eqs. (4) and (5) are given below;

Theorem 1 Assume that $W(k), U(k)$ and $V(k)$, are the differential transforms of the functions $w(t), u(t)$ and $v(t)$, respectively, then

(1-a) If $w(t)=u(t) \pm v(t)$, then $W(k)=U(k) \pm V(k)$.

(1-b) If $w(t)=\lambda u(t)$, then $W(k)=\lambda U(k)$.

(1-c) If $w(t)=\frac{d^{m} u(t)}{d t^{m}}$, then $W(k)=\frac{(k+m) !}{k !} U(k+m)$.

(1-d) If $w(t)=u(t) v(t)$, then $W(k)=\sum_{\ell=0}^{k} U(\ell) V(k-\ell)$.

(1-e) If $w(x)=x^{m}$ then $W(k)=\delta(k-m)=\left(\begin{array}{ll}1 & k=m, \\ 0 & \text { otherwise }\end{array}\right.$

(1-f) If $w(t)=u(t+a)$, then $W(k)=\sum_{\ell=k}^{N}\left(\begin{array}{l}\ell \\ k\end{array}\right) a^{\ell-k} U(\ell)$, for $N \rightarrow \infty$.

$(1-\mathrm{g})$ If $w(t)=\frac{d^{n}}{d t^{n}}[u(t+a)]$, then $W(k)=\frac{(k+n) !}{k !} \sum_{\ell=k+n}^{N}\left(\begin{array}{l}\ell \\ k+n\end{array}\right) a^{\ell-k-n} U(\ell)$, for $N \rightarrow \infty$.

Proof. See ([26], and their references).

Now we state the fundamental theorem of this paper.

Theorem 2. Assume that $\boldsymbol{W}(\boldsymbol{k}), \boldsymbol{U}_{\mathbf{1}}(\boldsymbol{k})$ and $\boldsymbol{U}_{\mathbf{2}}(\boldsymbol{k})$, are the differential transforms of the functions $\boldsymbol{w}(\boldsymbol{t}), \boldsymbol{u}_{\mathbf{1}}\left(\boldsymbol{q}_{\mathbf{1}} \boldsymbol{t}\right)$ and $\boldsymbol{u}_{\mathbf{2}}\left(\boldsymbol{q}_{\mathbf{2}} \boldsymbol{t}\right)$, respectively, and $\boldsymbol{q}_{\mathbf{1}}, \boldsymbol{q}_{\mathbf{1}} \in(\mathbf{0}, \mathbf{1})$, then

(2-a) If $w(t)=u_{1}\left(q_{1} t\right) u_{2}\left(q_{2} t\right)$, then $W(k)=\sum_{\ell=0}^{k} q_{1}^{\ell} q_{2}^{k-\ell} U_{1}(\ell) U_{2}(k-\ell)$.

(2-b) If $w(t)=u(q t) v(t-\tau)$, then for $N \rightarrow \infty$

$$
W(k)=\sum_{\ell=0}^{k} \sum_{s=k-\ell}^{N}\left(\begin{array}{l}
s \\
k-\ell
\end{array}\right) q^{\ell}(-\tau)^{s-k+\ell} U(\ell) V(s) .
$$

(2-c) If $w(t)=u_{1}\left(t-\tau_{1}\right) u_{2}\left(t-\tau_{2}\right)$, then for $N \rightarrow \infty$

$$
W(k)=\sum_{\ell=0}^{k}\left[\left(\sum_{s_{1}=\ell}^{N}\left(\begin{array}{l}
s_{1} \\
\ell
\end{array}\right)\left(-\tau_{1}\right)^{s_{1}-\ell} U_{1}\left(s_{1}\right)\right)\left(\sum_{s_{2}=k-\ell}^{N}\left(\begin{array}{l}
s_{2} \\
k-\ell
\end{array}\right)\left(-\tau_{2}\right)^{s_{2}-k+\ell} U_{2}\left(s_{2}\right)\right)\right] .
$$

Proof. (2-a) By using Leibnitz formula, we get 


$$
\frac{d^{k} w(t)}{d t^{k}}=\frac{d^{k}}{d t^{k}}\left[u_{1}\left(q_{1} t\right) u_{2}\left(q_{2} t\right)\right]=\sum_{\ell=0}^{k}\left(\begin{array}{c}
k \\
\ell
\end{array}\right) q_{1}^{\ell} \frac{d^{\ell}}{d \tilde{t}^{\ell}} u_{1}(\tilde{t}) q_{2}^{k-\ell} \frac{d^{k-\ell}}{d \hat{t}^{k-\ell}} u_{2}(\hat{t}),
$$

where $\hat{t}=q_{1} t$, and $\tilde{t}=q_{1} t$, therefore

$$
\left[\frac{d^{k} w(t)}{d t^{k}}\right]_{t=t_{0}}=\sum_{\ell=0}^{k}\left(\begin{array}{l}
k \\
\ell
\end{array}\right)\left[q_{1}^{\ell} \ell ! U_{1}(\ell)\right]\left[q_{2}^{k-\ell}(k-\ell) ! U_{2}(k-\ell)\right]=\sum_{l=0}^{k} k ! q_{1}^{\ell} q_{2}^{k-\ell} U_{1}(\ell) U_{2}(k-\ell),
$$

then from (6), we get

$$
W(k)=\frac{1}{k !}\left[\frac{d^{k} w(t)}{d t^{k}}\right]_{t=t_{0}}=\sum_{\ell=0}^{k} q_{1}^{\ell} q_{2}^{k-\ell} U_{1}(\ell) U_{2}(k-\ell) . \text { where } k=0,1, \ldots, \infty
$$

(2-b) Analogously from to previous Theorems we get

$$
\frac{d^{k}}{d t^{k}} w(t)=\frac{d^{k}}{d t^{k}}[u(q t) v(t-\tau)]=\sum_{\ell=0}^{k}\left(\begin{array}{l}
k \\
\ell
\end{array}\right) q^{\ell} \frac{d^{\ell}}{d \tilde{t}^{\ell}} u(\tilde{t}) \frac{d^{k-\ell}}{d t^{k-\ell}} v(t-\tau),
$$

where $\tilde{t}=q t$, therefore

$$
\left[\frac{d^{k}}{d t^{k}} w(t)\right]_{t=t_{0}}=\sum_{\ell=0}^{k}\left(\begin{array}{l}
k \\
\ell
\end{array}\right) q^{\ell} \ell !(k-\ell) ! U(\ell) V(k-\ell)=\sum_{\ell=0}^{k} k ! q^{\ell} U(\ell) V(k-\ell),
$$

where from Theorem 1-f:

$$
V(k-\ell)=\sum_{s=k-\ell}^{N}\left(\begin{array}{l}
S \\
k-\ell
\end{array}\right)(-\tau)^{s-k+\ell} V(s) .
$$

By substituting the (7) in (6), we get

$$
\left[\frac{d^{k}}{d t^{k}} w(t)\right]_{t=t_{0}}=k ! \sum_{\ell=0}^{k} \sum_{s=k-\ell}^{N}\left(\begin{array}{l}
s \\
k-\ell
\end{array}\right) q^{\ell}(-\tau)^{s-k+\ell} U(\ell) V(s),
$$

and therefore substituting these value in (4), the proof of (2-b) is completed.

(2-c) Analogously to part (2-a), we have

$$
\frac{d^{k}}{d t^{k}} w(t)=\frac{d^{k}}{d t^{k}}\left[u_{1}\left(t-\tau_{1}\right) u_{2}\left(t-\tau_{2}\right)\right]=\sum_{\ell=0}^{k}\left(\begin{array}{l}
k \\
\ell
\end{array}\right) \frac{d^{\ell}}{d t^{\ell}} u_{1}\left(t-\tau_{1}\right) \frac{d^{k-\ell}}{d t^{k-\ell}} u_{2}\left(t-\tau_{2}\right),
$$

therefore

$$
\left[\frac{d^{k}}{d t^{k}} w(t)\right]_{t=t_{0}}=\sum_{\ell=0}^{k}\left(\begin{array}{c}
k \\
\ell
\end{array}\right) \ell !(k-\ell) ! \mathcal{U}_{1}(\ell) \mathcal{U}_{2}(k-\ell)=k ! \sum_{\ell=0}^{k} \mathcal{u}_{1}(\ell) \mathcal{U}_{2}(k-\ell)
$$

where

$$
U_{1}(\ell)=\sum_{s_{1}=\ell}^{N}\left(\begin{array}{c}
s_{1} \\
\ell
\end{array}\right)\left(-\tau_{1}\right)^{s_{1}-\ell} U_{1}\left(s_{1}\right) \text {, and } U_{2}(k-\ell)=\sum_{s_{2}=k-\ell}^{N}\left(\begin{array}{l}
s_{2} \\
k-\ell
\end{array}\right)\left(-\tau_{2}\right)^{s_{2}-k+\ell} U_{2}\left(s_{2}\right) .
$$

by substituting these value in (8), and using (4), the result of (3-b) is obtained, and therefore the proof is completed.

Theorem 3. Assume that $p, q \in(0,1)$, and $\tau_{1}, \tau_{2}>0$ then for $k=1,2, \ldots, N$,

(3-a) If $w(t)=\int_{t_{0}}^{t} v_{1}(q s) v_{2}\left(s-\tau_{2}\right) d s$, then for $N \rightarrow \infty$

$$
W(k)=\frac{1}{k} \sum_{\ell=0}^{k-1} \sum_{s=k-\ell-1}^{N}\left(\begin{array}{l}
s \\
k-\ell-1
\end{array}\right) q^{\ell}\left(-\tau_{2}\right)^{s-k+\ell+1} V_{1}(\ell) V_{2}(s),
$$

(3-b) If $w(t)=u(p t) \int_{t_{0}}^{t} v_{1}(q s) v_{2}\left(s-\tau_{2}\right) d s$, then for $N \rightarrow \infty$ 


$$
W(k)=\sum_{\ell=0}^{k} \sum_{\ell_{0}=0}^{k-\ell-1} \sum_{s=k-\ell-\ell_{0}-1}^{N}\left(\begin{array}{l}
s \\
k-\ell-\ell_{0}-1
\end{array}\right) \frac{1}{k-\ell} p^{\ell} q^{\ell_{0}}\left(-\tau_{2}\right)^{s-k+\ell+\ell_{0}+1} U(\ell) V_{1}\left(\ell_{0}\right) V_{2}(s) .
$$

(3-c) If $w(t)=u\left(t-\tau_{1}\right) \int_{t_{0}}^{t} v_{1}(q s) v_{2}\left(s-\tau_{2}\right) d s$, then $W(k)=\sum_{\ell=0}^{k} u(\ell) \mathcal{Y}(k-\ell)$, where for $N \rightarrow \infty$,

$$
\begin{aligned}
& u(\ell)=\sum_{s_{0}=\ell}^{N}\left(\begin{array}{l}
s_{0} \\
\ell
\end{array}\right)\left(-\tau_{1}\right)^{s_{0}-\ell} U\left(s_{0}\right) . \\
& y(k-\ell)=\frac{1}{k-\ell} \sum_{\ell_{0}=0}^{k-\ell-1} \sum_{s=k-\ell-\ell_{0}-1}^{N}\left(\begin{array}{l}
s \\
k-\ell-\ell_{0}-1
\end{array}\right) q^{\ell_{0}}\left(-\tau_{2}\right)^{s-k+\ell+\ell_{0}+1} V_{1}\left(\ell_{0}\right) V_{2}(s) .
\end{aligned}
$$

Proof. (3-a) Analogously, for $k=1,2, \ldots, N$, we have

$$
\frac{d^{k}}{d t^{k}} w(t)=\frac{d^{k-1}}{d t^{k-1}}\left[v_{1}(q t) v_{2}\left(t-\tau_{2}\right)\right]=\sum_{\ell=0}^{k-1}\left(\begin{array}{l}
k-1 \\
\ell
\end{array}\right) q^{\ell} \frac{d^{\ell}}{d \hat{t}^{\ell}} v_{1}(\hat{t}) \frac{d^{k-\ell-1}}{d t^{k-\ell-1}} v_{2}\left(t-\tau_{2}\right),
$$

where $\hat{t}=q t$, therefore

$$
\left[\frac{d^{k}}{d t^{k}} w(t)\right]_{t=t_{0}}=\sum_{\ell=0}^{k-1}\left(\begin{array}{l}
k-1 \\
\ell
\end{array}\right) q^{\ell} \ell ! V_{1}(\ell)(k-\ell-1) ! V_{2}(k-\ell-1),
$$

where using Theorem. 1-(f)

$$
\mathcal{V}_{2}(k-\ell-1)=\sum_{s=k-\ell-1}^{N}\left(\begin{array}{l}
S \\
k-\ell-1
\end{array}\right)\left(-\tau_{2}\right)^{s-k+\ell+1} V_{2}(s) .
$$

by substituting (10) in (9), and using (4), we get

$$
W(k)=\frac{1}{k !}\left[\frac{d^{k}}{d t^{k}} w(t)\right]_{t=t_{0}} \quad=\frac{1}{k} \sum_{\ell=0}^{k-1} \sum_{s=k-\ell-1}^{N}\left(\begin{array}{l}
s \\
k-\ell-1
\end{array}\right) q^{\ell}\left(-\tau_{2}\right)^{s-k+\ell+1} V_{1}(\ell) V_{2}(s),
$$

and therefore the result of (4-a) is obtained.

(3-b) Let $y(t)=\int_{t_{0}}^{t} v_{1}(q s) v_{2}\left(s-\tau_{2}\right) d s$, then

$$
\frac{d^{k}}{d t^{k}} w(t)=\frac{d^{k}}{d t^{k}}[u(p t) y(t)]=\sum_{\ell=0}^{k}\left(\begin{array}{l}
k \\
\ell
\end{array}\right) p^{\ell} \frac{d^{\ell}}{d \hat{t}^{\ell}} u(\hat{t}) \frac{d^{k-\ell}}{d t^{k-\ell}} y(t),
$$

where $\hat{t}=p t$, therefore

$$
\left[\frac{d^{k}}{d t^{k}} w(t)\right]_{t=t_{0}}=\sum_{\ell=0}^{k}\left(\begin{array}{l}
k \\
\ell
\end{array}\right) p^{\ell} \ell ! U(\ell)(k-\ell) ! y(k-\ell),
$$

where using previous part (4-a), we have

$$
y(k-\ell)=\frac{1}{k-\ell} \sum_{\ell_{0}=0}^{k-\ell-1} \sum_{s=k-\ell-\ell_{0}-1}^{N}\left(\begin{array}{l}
S \\
k-\ell-\ell_{0}-1
\end{array}\right) q^{\ell_{0}}\left(-\tau_{2}\right)^{s-k+\ell+\ell_{0}+1} V_{1}\left(\ell_{0}\right) V_{2}(s),
$$

by substituting (12) in (11), and using (4), we get

$$
W(k)=\sum_{\ell=0}^{k} \sum_{\ell_{0}=0}^{k-\ell-1} \sum_{s=k-\ell-\ell_{0}-1}^{N}\left(\begin{array}{l}
s \\
k-\ell-\ell_{0}-1
\end{array}\right) \frac{1}{k-\ell} p^{\ell} q^{\ell_{0}}\left(-\tau_{2}\right)^{s-k+\ell+\ell_{0}+1} U(\ell) V_{1}\left(\ell_{0}\right) V_{2}(s) .
$$

(3-c) Similar on (4-b), let $y(t)=\int_{t_{0}}^{t} v_{1}(q s) v_{2}\left(s-\tau_{2}\right) d s$, then

$$
\frac{d^{k}}{d t^{k}} w(t)=\frac{d^{k}}{d t^{k}}\left[u\left(t-\tau_{1}\right) y(t)\right]=\sum_{\ell=0}^{k}\left(\begin{array}{l}
k \\
\ell
\end{array}\right) \frac{d^{\ell}}{d t^{\ell}} u\left(t-\tau_{1}\right) \frac{d^{k-\ell}}{d t^{k-\ell}} y(t),
$$

then 


$$
\left[\frac{d^{k}}{d t^{k}} w(t)\right]_{t=t_{0}}=\sum_{\ell=0}^{k}\left(\begin{array}{c}
k \\
\ell
\end{array}\right) \ell !(k-\ell) ! U(\ell) y(k-\ell),
$$

therefore $W(k)=\sum_{\ell=0}^{k} U(\ell) \mathcal{Y}(k-\ell)$, where $U(\ell)$ obtained from Theorem. (1-f) as follow

$$
u(\ell)=\sum_{s_{0}=\ell}^{N}\left(\begin{array}{c}
s_{0} \\
\ell
\end{array}\right)\left(-\tau_{1}\right)^{s_{0}-\ell} U\left(s_{0}\right),
$$

and $\mathcal{Y}(k-\ell)$ is same as (12), and therefore the proof is completed.

\section{Applications and Numerical Examples}

In this section, we present the prototype examples to clarify the accuracy of the presented method. In these examples, we first obtain a recurrence relation for the differential transform of an integral equation and solve it by programming in MAPLE environment. These prototype examples are chosen such that there exist exact solutions for them.

Example 1. In the first example, consider the following non-linear integro-differential equation with multi

proportional delays

$$
u^{\prime}(t) u(t)-u\left(\frac{t}{2}\right)-\frac{3}{2} u\left(\frac{t}{2}\right) \int_{0}^{t} u(s) u\left(\frac{s}{2}\right) d s=0, t \geq 0,
$$

subject to initial condition $u(0)=1$.

From initial condition, we get $u^{\prime}(0)-1=0$, then $u^{\prime}(0)=1$, therefore differential transform version of initial conditions $u(0)=1, u^{\prime}(0)=1$ are $U(0)=1, U(1)=1$ respectively, and the differential transform version of Eq. (13) for $k=1,2, \ldots, N$ is

$$
\sum_{\ell=0}^{k-1}(\ell+1) U(\ell+1) U(k-\ell)-\left(\frac{1}{2}\right)^{k} U(k)-\frac{3}{2} \sum_{\ell=0}^{k-1} \sum_{s=0}^{k-\ell-1} \frac{1}{k-\ell}\left(\frac{1}{2}\right)^{k-s-1} U(\ell) U(s) U(k-\ell-s-1)=0,
$$

where $U(k)$ is the differential transform of $u(t)$.

Using Eqs.(14), by taking $N=5$, the following system is obtained:

$$
\begin{aligned}
& 2 U(2)-1=0 \\
& \frac{11}{4} U(2)+3 U(3)-\frac{15}{8}=0 \\
& \frac{31}{8} U(3)+2 U(2)^{2}+4 U(4)-\frac{13}{16}-U(2)=0 \\
& \frac{79}{16} U(4)+5 U(2) U(3)+5 U(5)-\frac{39}{64} U(3)-\frac{7}{8} U(2)-\frac{1}{8}=0, \\
& \frac{191}{32} U(5)+6 U(2) U(4)+3 U(3)^{2}+6 U(6)-\frac{33}{80} U(4)-\frac{69}{128} U(3)-\frac{37}{160} U(2)^{2}-\frac{13}{64} U(2)=0,
\end{aligned}
$$

Solving the above system and using the inverse transformation rule (5), we get the following series solution

$$
u(t)=1+t+\frac{1}{2} t^{2}+\frac{1}{6} t^{3}+\frac{1}{24} t^{4}+\frac{1}{120} t^{5} .
$$

Note that when $N \rightarrow \infty$ by solving the obtained system, we get the following series solution

$$
u(t)=1+t+\frac{1}{2 !} t^{2}+\frac{1}{3 !} t^{3}+\frac{1}{4 !} t^{4}+\ldots+\frac{1}{N !} t^{N} .
$$

The closed form of above series solution is $u(t)=e^{t}$, which is the exact solution of Eq. (13).

Example 2. In the second example, consider the following nonlinear delay integro-differential equation

$$
\begin{gathered}
u^{\prime}(t)-u(t-1)-\frac{8}{3} u\left(t-\frac{1}{2}\right) \int_{0}^{t} u(s-1) d s=t-\frac{10}{3} t^{3}+ \\
\frac{4}{3} t^{4}-\frac{8}{9} t^{5}, t \geq 0,
\end{gathered}
$$

subject to initial condition $u(t)=1$, for $t \in\left[-\frac{1}{2}, 0\right]$.

From initial condition, we get $u(0)=1$, therefore by substituted $t=0$, in Eq.(16) we get

$$
u^{\prime}(0)-u(-1)=0,
$$

Similar on previous example, the differential transform version of Eq. (16) for $k=1,2, \ldots, M$ and for $N \rightarrow \infty$ is

$$
\begin{aligned}
& (k+1) U(k+1)-\sum_{\ell=k}^{N}\left(\begin{array}{l}
\ell \\
k
\end{array}\right)(-1)^{\ell-k} U(\ell)-\frac{8}{3} \sum_{\ell=0}^{k-1} \sum_{s_{0}=\ell}^{N} \sum_{s=k-\ell-1}^{N}\left(\begin{array}{l}
S_{0} \\
\ell
\end{array}\right)\left(\begin{array}{l}
S \\
k-\ell-1
\end{array}\right)\left(-\frac{1}{2}\right)^{s_{0}-\ell}(-1)^{s-k+\ell+1} U\left(s_{0}\right) U(s) \\
& =\delta(k-1)-\frac{10}{3} \delta(k-3)+\frac{4}{3} \delta(k-4)-\frac{8}{9} \delta(k-5),
\end{aligned}
$$

and the differential version of initial condition (17) is

$$
U(1)-\sum_{k=0}^{M}(-1)^{k} U(k)=0,
$$

where $U(k)$ is the differential transform of $u(t)$.

Using Eqs.(17), by taking $M=5$, and $N=3$, the following system is obtained: 


$$
\begin{aligned}
& \frac{2}{3} U(2)+3 U(1)-\frac{11}{3}-\frac{4}{3} U(1)^{2}+2 U(1) U(2)-\frac{5}{3} U(1) U(3)-\frac{2}{3} U(2)^{2}+U(2) U(3)-\frac{1}{3} U(3)^{2}=0, \\
& \frac{13}{3} U(2)-4 U(1)+\frac{10}{3} U(1)^{2}-7 U(1) U(2)+\frac{41}{6} U(1) U(3)+\frac{10}{3} U(2)^{2}-6 U(2) U(3)+\frac{5}{2} U(3)^{2}=0, \\
& 4 U(4)+\frac{17}{3} U(3)-\frac{32}{9} U(2)+\frac{64}{9} U(1) U(2)-\frac{31}{3} U(1) U(3)-\frac{50}{9} U(2)^{2}+\frac{121}{9} U(2) U(3)-\frac{22}{3} U(3)^{2}-\frac{4}{3} U(1)^{2}+\frac{10}{3}=0, \\
& 5 U(5)-v \frac{10}{3} U(3)+\frac{23}{3} U(1) U(3)-\frac{85}{6} U(2) U(3)+\frac{43}{4} U(3)^{2}-\frac{20}{9} U(1) U(2)+\frac{32}{9} U(2)^{2}-\frac{4}{3}=0, \\
& 6 U(6)-2 U(1) U(3)+\frac{22}{3} U(2) U(3)-\frac{17}{2} U(3)^{2}-\frac{8}{9} U(2)^{2}+\frac{8}{9}=0,
\end{aligned}
$$

and using (19), we get

$$
2 U(1)-U(2)+U(3)-U(4)+U(5)-1=0,
$$

Solving the system (20) and differential transform version of initial condition (19), simultaneously, and using the inverse transformation rule (5), we get the following series solution

$$
u(t)=1+t+t^{2} \text {. }
$$

Note that for $M>5$ and $N \rightarrow \infty$ we evaluate the same solution, which is the exact solution of Eq.(16) with the initial conditions (17).

Example 3. Finally, consider the following nonlinear delay integro-differential equation

$$
\begin{aligned}
& \sum_{\ell=0}^{k}(\ell+1) U(\ell+1) U(k-\ell)+\sum_{\ell=k}^{N}\left(\begin{array}{l}
\ell \\
k
\end{array}\right)\left(-\frac{1}{2}\right)^{\ell-k} U(\ell)-\frac{4}{k} \sum_{\ell=0}^{k-1} \sum_{s=k-\ell-1}^{N}\left(\begin{array}{l}
s \\
k-\ell-1
\end{array}\right)\left(-\frac{1}{2}\right)^{s-k+\ell+1} U(\ell) U(s) \\
& =\frac{5}{4} \delta(k)-\frac{17}{2} \delta(k-2)+\delta(k-3)+3 \delta(k-4)-\frac{5}{4} \delta(k-5),
\end{aligned}
$$

and the differential version of initial condition (23) is

$$
U(1)-\sum_{k=0}^{M}\left(-\frac{1}{2}\right)^{k} U(k)=\frac{5}{4},
$$

where $U(k)$ is the differential transform of $u(t)$.

Using Eqs.(23), by taking $M=5$, and $N=3$, the following system is obtained:

$$
\begin{aligned}
& U(1)^{2}+3 U(1)+\frac{5}{4} U(3)-\frac{3}{4} U(4)-4=0 \\
& \frac{5}{2} U(1) U(2)+3 U(2)+\frac{5}{2} U(4)-4 U(1)+U(1)^{2}+\frac{1}{4} U(1) U(3)-\frac{1}{8} U(1) U(4)+\frac{17}{2}=0 \\
& 3 U(1) U(3)+\frac{5}{3} U(2)^{2}+3 U(3)-\frac{8}{3} U(2)-\frac{4}{3} U(1)^{2}+2 U(1) U(2)+\frac{2}{3} U(1) U(4)+\frac{1}{6} U(2) U(3) \\
& -\frac{1}{12} U(2) U(4)-1=0 \\
& \frac{7}{2} U(1) U(4)+4 U(2) U(3)+5 U(5)+3 U(4)-2 U(3)-2 U(1) U(2)+2 U(1) U(3)+U(2)^{2} \\
& +\frac{1}{2} U(2) U(4)+\frac{1}{8} U(3)^{2}-\frac{1}{16} U(3) U(4)-3=0, \\
& 6 U(1) U(5)+\frac{23}{5} U(2) U(4)+\frac{12}{5} U(3)^{2}+6 U(6)-\frac{8}{5} U(4)-\frac{8}{5} U(1) U(3)+2 U(1) U(4)-\frac{4}{5} U(2)^{2} \\
& +2 U(2) U(3)+\frac{1}{2} U(3) U(4)-\frac{1}{20} U(4)^{2}+\frac{4}{5}=0,
\end{aligned}
$$

and using (25), we get

conditions (23).

$\frac{1}{2} U(1)+\frac{1}{4} U(2)-\frac{1}{8} U(3)+\frac{1}{16} U(4)-\frac{1}{32} U(5)-\frac{1}{4}=0$,

Solving the system (26) and differential transform version of initial condition (25), simultaneously, and using the inverse transformation rule (5), we get the following series solution

$$
u(t)=1+t-t^{2} .
$$

Note that for $M>5$ and $N \rightarrow \infty$ we evaluate the same solution, which is the exact solution of Eq.(22) with the initial

\section{Conclusions}

In this paper, we have shown that the differential transformation method can be used successfully for solving the nonlinear delay integro-differential equations. New theorems were presented with their proofs and as application some prototype examples were carried out. It is worth noting that DTM does not require complex computational work such as Adomian polynomials in Adomian decomposition and 
Lagrange multipliers by solving stationary equations in variational iteration method.

\section{References}

[1] M. M. Kabir, A. Borhanifar and R. Abazari, Application of $\left(\frac{G \prime}{G}\right)$-expansion method to Regularized Long Wave (RLW) equation, Computers and Mathematics with Applications 61 (8) (1991), 233-241.

[2] A. Borhanifar, A. Zamiri, Application of the $\left(\frac{G^{\prime}}{G}\right)$-expansion method for the Zhiber-Shabat equation and other related equations, Mathematical and Computer Modeling 54 (9-10) (2011), 2109-2116.

[3] A. Borhanifar, R. Abazari, General Solution of Generalized (2+1)-Dimensional Kadomtsev-Petviashvili (KP) Equation by Using the $\left(\frac{G \prime}{G}\right)$-expansion method, American Journal of computational Mathematics 1 (2011), 219-225.

[4] A. Borhanifar, M. M. Kabir and L. Maryam Vahdat, New periodic and soliton wave solutions for the generalized Zakharov system and $(2+1)$-dimensional Nizhnik-Novikov-Veselov system, Chaos, Solitons and Fractals 42 (2009), 1646-1654.

[5] A. Borhanifar, M. M. Kabir, New periodic and soliton solutions by application of Exp-function method for nonlinear evolution equations, Journal of Computational and Applied Mathematics 229 (2009), 158-167.

[6] H. Brunner, The numerical solution of neutral Volterra integro-differential equations with delay arguments, Ann. Numer. Math. 1 (1994), 309-322.

[7] H. Brunner, W. Zhang, Primary discontinuities in solutions for delay integro-differential equations, Methods Appl. Anal. 6 (1999), 525-533.

[8] H. Brunner, On the discretization of differential and Volterra integral equations with variable delay, BIT 37 (1997), 1-12.

[9] H. Brunner, The numerical analysis of functional integral and integro-differential equations of Volterra type, Acta Numerica. (2004), 55-145.

[10] H. Brunner, Q.-Y. Hu, Superconvergence of iterated collocation solutions for Volterra integral equations with variable delays, SIAM J. Numer. Anal. 43 (2005), 1934-1949.

[11] H. Brunner, Q.-Y. Hu, Optimal superconvergence results for delay integro-differential equations of pantograph type, SIAM J. Numer. Anal. 45 (2007), 986-1004.

[12] H. Brunner, Q.-Y. Hu, Q. Lin, Geometric meshes in collocation methods for Volterra integral equations with proportional delays, IMA J. Numer. Anal. 21 (2001), 783-798.

[13] H. Brunner, R. Vermiglio, Stability of solutions of neutral functional integro-differential equations and their discretizations, Computing 71 (2003), 229-245.

[14] H. Brunner, Recent advances in the numerical analysis of Volterra functional differential equations with variable delays, J. Comput. Appl. Math. 228 (2009), 524-537.

[15] C. T. H. Baker, N. J. Ford, Asymptotic error expansions for linear multistep methods for a class of delay integro-differential equations, Bull. Soc. Math. Greece (N. S.) 31 (1990), 5-18.

[16] C. T. H. Baker, N. J. Ford, Stability properties of a scheme for the approximate solution of a delay-integro-differential equation, Appl. Numer. Math. 9 (1992), 357-370.

[17] T. Koto, Stability of Runge-Kutta methods for delay integro-differential equations, J. Comput. Appl. Math. 145 (2002), 483-492.

[18] T. Koto, Stability of $\theta$-methods for delay integro-differential equations, J. Comput. Appl. Math. 161 (2003), 393-404.

[19] W. H. Enright, M. Hu, Continous Runge-Kutta methods for neutral Volterra integro-differential equations with delay, Appl. Numer. Math. 24 (1997), 175-190.

[20] A. Borhanifar, R. Abazari, Numerical study of nonlinear Schrodinger and coupled Schrodinger equations by differential transformation method, Optics Communications 283 (2010), 2026-2031.

[21] R. Abazari, A. Borhanifar, Numerical study of the solution of the Burgers and coupled Burgers equations by a differential transformation method, Computers and Mathematics with Applications 59 (2010), 2711-2722.

[22] J. K. Zhou, Differential Transformation and its Application for Electrical Circuits, Huazhong University Press, Wuhan, China, 1986.

[23] Fatma Ayaz, Solutions of the system of differential equations by differential transform method, Appl. Math. Comput. 147 (2004), 547-567.

[24] M. Mossa Al-Sawalha, M. S. M. Noorani, Application of the differential transformation method for the solution of the hyperchaotic Rossler system, Communications in Nonlinear Science and Numerical Simulation 14 (2009), 1509-1514.

[25] M. Mossa Al-Sawalha, M. S. M. Noorani, A numeric-analytic method for approximating the chaotic Chen system, Chaos, Solitons \& Fractals, 42 (2009), 1784-1791.

[26] F. Ayaz, Application of differential transform method to differential-algebraic equations, Appl. Math. Comput. 152 (2004), 649-657.

[27] A. Arikoglu, I. Ozkol, Solution of difference equations by using differential transform method, Appl. Math. Comput. 174 (2006), 1216-1228.

[28] A. Arikoglu, I. Ozkol, Solution of differential-difference equations by using differential transform method, Appl. Math. Comput. 181 (2006), 153-162.

[29] F. Kangalgil, F. Ayaz, Solitary wave solutions for the KdV and $m K d V$ equations by differential transform method, Chaos, Solitons \& Fractals 41 (2009), 464-472.

[30] C. K. Chen, Solving partial differential equations by two dimensional differential transform, Appl. Math. Comput. 106 (1999), 171-179.

[31] M. J. Jang, C. L. Chen, Y. C. Liy, Two-dimensional differential transform for Partial differential equations, Appl. Math. Comput. 121 (2001), 261-270.

[32] A. Kurnaz, G. Oturanc, ME. Kiris, $n$-Dimensional differential transformation method for solving linear and nonlinear PDE's, Int J Comput Math 82 (2005), 369-380. 
[33] M. O. Al-Amr, New applications of reduced differential transform method, Alexandria Engineering Journal 53 (2014), 243-247.

[34] S. Momani, Z. Odibat, I. Hashim, Algorithms for nonlinear fractional partial differential equations: A selection of numerical methods, Topological method in Nonlinear Analysis 31 (2008), 211-226.

[35] A. Arikoglu, I. Ozkol, Solution of fractional differential equations by using differential transform method, Chaos Soliton. Fract. 34 (2007), 1473-1481.

[36] A. letinkaya, O. Kiymaz, The solution of the time-fractional diffusion equation by the generalized differential transform method, Mathematical and Computer Modelling 57 (2013), $2349-2354$

[37] Y. Keskin, A. Kurnaz, M. E. Kiris, G. Oturanc, Approximate solutions of generalized pantograph equations by the differential transform method, Int. J. Nonlinear Sci. 8 (2007), 159-164.

[38] S. M. Abdelghany, K. M. Ewis, A. A. Mahmoud, Mohamed M. Nassar, Vibration of a circular beam with variable cross sections using differential transformation method, Beni - suef university journal of basic and applied sciences 4 (2015), 185-191.

[39] R. Lal, N. Ahlawat, Axisymmetric vibrations and buckling analysis of functionally graded circular plates via differential transform method, European Journal of Mechanics / A Solids (in press). DOI: 10.1016/j.euromechsol. 2015. 02.004

[40] A. M. A. El-Sayed, H. M. Nour, W. E. Raslan, E. S. El-Shazly, A study of projectile motion in a quadratic resistant medium via fractional differential transform method, Appl. Math. Modelling (in press). DOI: 10.1016/j.apm.2014.10.018

[41] A. Arikoglu, I. Ozkol, Solution of boundary value problems for integro-differential equations by using differential transform method, Appl. Math. Comput. 168 (2005), 1145-1158.

[42] Z. M. Odibat, Differential transform method for solving Volterra integral equations with separable kernels, Math. Comput. Model. 48 (7-8) (2008), 1144-1149.

[43] A. Arikoglu, I. Ozkol, Solutions of integral and integro-differential equation systems by using differential transform method, Computers and Mathematics with Applications 56 (2008), 2411-2417. 